\title{
Le Tan et le Temps
}

Changements techniques et dimension historique du tannage en France (XIVe-XVIIIe siècles)

\section{Eva Halasz-Csiba}

\section{(2) OpenEdition}

1 Journals

Édition électronique

URL : https://journals.openedition.org/tc/1585

DOI : $10.4000 /$ tc. 1585

ISSN : 1952-420X

Éditeur

Éditions de l'EHESS

\section{Édition imprimée}

Date de publication : 1 mars 2002

ISSN : 0248-6016

\section{Référence électronique}

Eva Halasz-Csiba, "Le Tan et le Temps », Techniques \& Culture [En ligne], 38 | 2002, mis en ligne le 04 septembre 2006, consulté le 29 septembre 2022. URL : http://journals.openedition.org/tc/1585; DOI : https://doi.org/10.4000/tc. 1585

Ce document a été généré automatiquement le 29 septembre 2022.

Tous droits réservés 


\section{Le Tan et le Temps}

Changements techniques et dimension historique du tannage en France (XIVe-XVIIIe siècles)

\section{Eva Halasz-Csiba}

\section{C'est un des thèmes techniques les plus diffus et il est assez curieux de constater qu'il n'ait encore jamais servi à édifier de théorie d'ensemble. \\ A. Leroi-Gourhan $(1971: 242)$}

1 Bien que personne ne puisse contester que la transformation de la peau en cuir fasse partie d'un «savoir technique universel » et que les cuirs et peaux aient constitué des matériaux de base indispensables et multiformes dans la plupart des civilisations, les études systématiques sur ce sujet sont rares et accusent jusqu'à nos jours des lacunes considérables.

On se sent perplexe devant ce paradoxe : l'importance du rôle que le cuir a joué dans la civilisation matérielle n'est nullement remise en cause et cependant, la place qu'on lui accorde -à mesurer en volume de recherche dans les diverses disciplines- reste étonnamment modeste (sinon inexistante) au regard des autres matériaux et techniques. Même les grands ouvrages de référence, tels que l'Histoire générale des techniques en quatre volumes (Daumas 1965), n'ont pas considéré la transformation de la peau en cuir comme un problème technique majeur dans les civilisations; c'est du moins ce que suggèrent la présentation très succincte (quelques pages à peine sur deux milles) consacrée à l'industrie du cuir et le manque quasi total d'analyses techniques. Situation inconfortable qui n'encourage pas à entreprendre des études de synthèse nécessairement interdisciplinaires, étant donné qu'elles auraient à tenir compte d'aspects variés (historiques, économiques, technologiques, sociaux...) qu'il faudrait examiner pour situer avec exactitude les changements techniques dans leur dimension historique.

3 Dans cet article, nous avons choisi d'étudier une période longue (et relativement peu documentée) qui va du XIVe au XVIIIe siècles, pour entamer un premier " défrichage » concernant les changements techniques dans le vaste domaine des différentes formes 
de tannage ${ }^{1}$ pratiquées en France. Cette période fut décisive du point de vue de l'évolution technique dans un contexte artisanal ${ }^{2}$.

4 Notre étude porte un intérêt particulier à la structure des savoirs techniques préindustriels dans le domaine des métiers transformateurs de la peau animale, afin qu'il soit possible de dégager et d'analyser les facteurs responsables des changements et des innovations.

$5 \mathrm{Si}$, au XXe siècle, l'industrie du cuir est devenue un secteur éminemment lié aux applications de la recherche menée dans des laboratoires spécialisés en chimie, le savoir des gens du métier -dans la période qui nous intéresse fut fondamentalement empirique, peu verbalisé, et généralement gardé secret. Un savoir technique d'ailleurs peu valorisé socialement, dans la mesure où le travail des tanneurs -transformateurs des dépouilles animales - touchait aux « tabous de la société médiévale (par exemple le sang, l'impureté, etc. ${ }^{3}$ ), et suscitait du mépris, voire des accusations contre ces pollueurs de rivière, surtout pendant les grandes épidémies.

6 À notre époque, les professionnels du cuir aiment à souligner, en parlant de leurs métiers respectifs, qu'il s'agit d'une véritable filière du cuir. De l'élevage jusqu'aux choix de la technologie du tannage et à la destination des cuirs et peaux (selon le mode de fabrication et les goûts de l'utilisateur), on passe par l'enchaînement de toute une série d'activités très diverses et cependant très liées, interdépendantes. Et chacune d'elles doit tenir compte de bon nombre de contraintes influant sur la qualité et la variété des produits finis.

7 En France, depuis la fondation des premières corporations (XIIe-XIVe siècles), l'industrie du cuir a souvent compté comme un "secteur-clé» dans l'ensemble de l'économie ${ }^{4}$, et cela pratiquement jusqu'au milieu du XXe siècle, en dépit du fait que cette industrie a longtemps gardé une structure artisanale ${ }^{5}$, avec de petites entreprises employant de un à cinq ouvriers, des installations très peu mécanisées et une technologie qui offrait souvent des aspects «archaïques» malgré les nombreux changements et innovations techniques intervenus depuis le XVIIIe siècle.

8 La devise des tanneurs français d'autrefois le souligne : «Pour avoir des bons cuirs, il faut du tan et du temps ». Sobre expression de l'essentiel s'il en est, et en même temps tout un programme professionnel qui désigne les vecteurs déterminants de la qualité des produits.

9 Cette formule caractérise fort bien ce que nous entreprenons ici, car elle évoque les notions-clés qui vont articuler notre analyse: le tan, ou les matériaux (agents) responsables du long processus de transformation des peaux vulnérables en un matériau de base résistant et durable, le cuir; le temps - dans au moins deux acceptions du terme, la durée et l'espace-temps- qui nous servira de fil conducteur.

La durée du tannage ${ }^{6}$ - «temps interne » du processus du travail-, excessivement longue et périlleuse, a constitué l'un des facteurs responsables de l'inertie technique pendant de longs siècles; puis ce problème capital a fini par déclencher l'application d'une série d'innovations pour entamer l'accélération et le raccourcissement du temps du travail. L'espace-temps situe notre étude principalement en France, pendant la longue période indiquée plus haut, avec deux époques saillantes : le tournant des XIVe et XVe siècles, et le XVIIIe siècle ${ }^{7}$.

Importance et variété des cuirs 
11 Peaux et pelleteries chaudes, souples, moelleuses, et cuirs solides et résistants, sont des matériaux omniprésents et indispensables de la vie quotidienne en France comme dans les autres pays européens entre le Moyen Âge et la fin du XIXe siècle. Il s'agit d'une matière première accessible, relativement peu coûteuse et surtout dotée de qualités incomparables, qui remplit de nombreuses fonctions vitales dans la société grâce à la richesse de ses propriétés.

12 Car ce matériau souple, en principe vulnérable, peut aussi devenir fort, ferme et particulièrement résistant comme les cuirs de fatigue qui fournissent les pièces de harnachement des chevaux et des autres bêtes de somme, et équipent aussi l'armée; ou bien comme le cuir bouilli médiéval, lequel, une fois moulé et martelé en forme de casque ou de bouclier, acquiert une dureté et une rigidité si exceptionnelles qu'il pourra arrêter les armes de métal. À la fois imperméables (à l'eau) et perméables (à l'air), incassables, légers, modelables, les récipients en cuir -outres, gourdes, seaux, sacs, boîtes - répondent aux besoins primordiaux de la conservation, du stockage et du transport des liquides (vin, huile, eau...) et des solides (aliments et objets les plus divers). Veloutées, chamoisées, soyeuses et colorées, les peaux douces et élastiques, taillées en vêtements ou même en sous-vêtements, lacets et ceintures, ou gants et chaussures, assurent confort et protection au corps humain. Réduite à une membrane mince, rigide, lisse et homogène, la peau transformée en parchemin est le support irremplaçable de l'écriture et de l'enluminure jusqu'à l'introduction massive du papier.

13 Cette étendue extrêmement vaste et inhabituellement contradictoire de qualités, rarement réunies dans un seul et même matériau, a su imposer le cuir pendant des siècles et des siècles dans presque tous les domaines de la vie matérielle. Habitat et habillement, transports, équipement militaire, objets domestiques, mobilier de luxe, utilisation dans l'agriculture puis applications industrielles -autant de grands secteurs avides de quantités considérables d'ouvrages en cuir ou en peau.

Les corps de métier

14 Pour satisfaire aux besoins toujours croissants et à des exigences de plus en plus pointues quant à la qualité fonctionnelle et esthétique des cuirs, un grand nombre de corps de métiers voient le jour à partir du XIIe siècle en France: Tanneurs, Baudroyeurs, Sueurs, Mégissiers et Boursiers sont parmi les premiers, mentionnés dès 1160 sous le règne de Louis VII; Pelletiers, Peaussiers, Parcheminiers, Corroyeurs, Cordouanniers et Basanniers sont réunis en corporations homologuées et fortement contrôlées dès le XIIIe siècle; tous sont transformateurs de la peau brute, mais différenciés selon le caractère spécifique de leurs productions.

15 Les petites peaux relativement faciles à manier et les fourrures seront apprêtées par les Mégissiers et les Pelletiers, puis parées, teintées et parfois même parfumées par la communauté des Peaussiers. Parchemins et vélins, ces peaux lisses, conservées sans aucun tannage, constituent l'œuvre des Parcheminiers.

16 Les grands cuirs lourds et épais, très difficiles à traiter, une fois transformés et pérennisés par les Tanneurs, doivent se faire "conroier » c'est-à-dire apprêter (« soigner » en langage médiéval), pour subir une finition conforme à leur destination, par des Corroyeurs, des Baudroyeurs ou éventuellement des Sueurs.

17 Enfin, les Cordouanniers ${ }^{8}$ et les Basanniers, deux corps de métiers liés principalement à la fabrication de la chaussure, ont le privilège de préparer eux-mêmes les cuirs spécifiques de leurs ouvrages: le cordouan et la basane. Ces deux matériaux, très 
appréciés et largement utilisés ${ }^{9}$ dès le XIIIe siècle, sont issus d'un emprunt technique précoce en provenance de la péninsule ibérique. Le cordouan, appelé aussi cuir de Cordoue, ouvre la longue liste des cuirs importés des pays lointains, abondamment cités dans les inventaires et comptes royaux: cuir de Cabes (Tunisie, 1153), cuir de Cappadoce (1250), de Damas (1302), d'Aragon (1380), de Syrie (1411), cuir de Hongrie (depuis 1380), ainsi que dans une période plus tardive, les maroquins ou cuirs du Maroc, les cuirs des Flandres, de Brabant, de Liège, de Russie, de Suède... une variété étonnante de matériaux répertoriés, dont on ne retrouve que de rares descriptions, soit à titre de produits de luxe ou de cadeaux diplomatiques, soit en tant qu'articles issus d'échanges commerciaux. En tout état de cause, il s'agit de matériaux indispensables, dont la présence de plus en plus affirmée à partir du milieu du XIIe siècle sert d'indicateur tangible pour mieux mesurer l'essor des échanges entre la France et le reste du monde ${ }^{10}$.

Si la multiplicité des contacts avec l'étranger a laissé des traces sans équivoque sur la dénomination des cuirs, il est bien plus difficile de déceler ses effets en matière d'emprunts techniques. Jusqu'au XVIIIe siècle, on ne dispose que de très peu de renseignements. Les exceptions sont d'autant plus significatives, comme nous le montrerons ultérieurement à travers l'examen du cuir de Cordoue et du cuir de Hongrie.

19 Malgré les lacunes considérables de la documentation, il nous a été possible de dégager quelques grandes lignes technologiques à travers le temps, grâce notamment aux données des diverses corporations. Leur analyse a permis de déterminer où se situaient les points de jonction qui ont généré des changements techniques dans le réseau bien ramifié des métiers du cuir ${ }^{11}$.

Principaux types de tannage et répartition géographique

20 Les procédés qui ont permis d'obtenir les divers cuirs et peaux sont trop variés pour qu'on puisse les réduire à un seul processus, le tannage proprement dit, dénomination que l'on devrait réserver au tannage végétal. Cette grande diversité technique nous mène au cœur de la problématique des tannages pratiqués par tel ou tel corps de métier, en révélant les principes de base sur lesquels repose la différenciation de leurs activités.

21 En tannant la peau, on la transforme par des actions physiques et chimiques en une substance qui résiste à la putréfaction et, plus ou moins, à l'eau, Elle ne reprendra plus son état initial de peau brute. On appelle cuir le produit de cette transformation. Le caractère irréversible du procédé constitue le signe distinctif et essentiel de toutes les techniques que l'on qualifie de véritable tannage ${ }^{12}$. Au cours de celui-ci, il s'agit de fixer différentes molécules dans la structure fibrillaire de la peau brute et de créer ainsi des liaisons de pontage stables entre les chaînes polypeptidiques du collagène et l'agent tannique.

On distingue trois principaux types de tannage : le tannage végétal, le tannage minéral et le tannage à la graisse. À côté de ces transformations véritables de la peau en cuir, il existe un certain nombre de techniques -qualifiées de pseudo-tannages ${ }^{13}-$, à l'issue desquelles les substances incorporées dans les interstices des fibres demeurent généralement solubles. Il ne s'agit donc pas d'un processus chimique irréversible, mais plutôt d'un certain type de conservation de la matière dont les effets restent réversibles sous des influences extérieures. 
23 En examinant les métiers du cuir pratiqués en France depuis l'époque médiévale, on peut constater qu'à l'exception des Parcheminiers, dont la technique n'entraîne pas de modification chimique de la peau, tous les autres métiers ont recours aux techniques du véritable tannage afin d'assurer une vaste gamme de produits résistants et durables. Pour saisir les principes qui structurent les grandes lignes technologiques dans le domaine du tannage, il faut par conséquent recourir à un autre fil conducteur que l'axe réversibilité-irréversibilité des processus.

«Dans cette masse universelle, des distinctions apparaîtront tout d'abord liées aux matériaux, au milieu zoologique ou social » (Leroi-Gourhan 1971 : 241). En définissant le tannage comme une interaction entre deux types de matière, les peaux animales et les agents tanniques, il devient possible de dégager le fil conducteur recherché dans la grande diversité des techniques en présence. L'extrême variété des peaux brutes, dont les spécificités (espèce, race, âge, sexe, mode de vie, etc., de l'animal) exigent des types de tannage différents, d'une part, et d'autre part la multiplicité des agents tanniques d'origine végétale, minérale ou animale, ainsi qu'un large éventail de matériaux auxiliaires, constituent les facteurs essentiels de la grande richesse de ce domaine. Et si le rôle déterminant de la nature de l'agent tannique a toujours été reconnu comme un facteur décisif de la qualité du cuir, les anciennes "recettes » de tannage nous prouvent que depuis l'Antiquité, les tanneurs ont recherché la combinaison d'effets de multiples matériaux pour améliorer ou diversifier les propriétés de leurs cuirs ${ }^{14}$.

Nous avons également jugé important de poser quelques jalons dans le domaine de la répartition géographique des principaux types de tannage. Notre objectif consiste à esquisser une grille de repères qui facilite l'identification des grandes lignes technologiques et permette de déceler plus aisément les mouvements et transformations consécutifs aux emprunts techniques.

Tannage végétal

26 En Europe, dans les zones forestières où abondent les cours d'eau, où le climat est humide et relativement froid, on vit se développer le véritable tannage végétal, pratiqué dans des fosses, en appliquant le tan (c'est-à-dire des écorces de chêne) comme principal agent tannique. Le pouvoir tannant de très nombreux végétaux a été reconnu depuis la plus haute Antiquité et extrait à cet effet des différentes parties de la plante (écorce, bois, racine, feuilles, fruits, gousses, galles, etc.).

Alors que le tannage lent, dans des fosses, des peaux lourdes et épaisses des bovins semble être une technique répandue en Europe médiévale, de nombreuses variantes techniques se sont diffusées à travers différentes contrées. Citons un exemple très particulier: le «tannage en sac» ou "en outre», technique archaïque et pourtant encore fréquente au XVIIIe siècle ${ }^{15}$, préférée à d'autres procédés pour sa simplicité et sa rapidité. Il s'agit de dépouiller un animal -surtout ovin ou caprin - en arrachant sa peau en entier (sans la couper) et de la remplir de décoctions de tanin végétal. Pour les animaux plus grands, on coud avec les peaux prises deux par deux une espèce de sac qui sera rempli de tanin. Ce procédé, qui dans sa forme primitive servait de toute évidence à fabriquer des outres, des flotteurs ou même des soufflets, a connu une variante technique au Maroc, associant le tannage en sac et le tannage dans les fosses, méthode de fabrication des fameux cuirs de luxe, les maroquins.

Tannage minéral

28 Les milieux géographiques, climatiques, botaniques, zoologiques, ont une influence incontestable, voire déterminante, sur le développement de tel ou tel type de tannage. 
Dans le climat chaud et sec de l'Asie Mineure et du Proche-Orient par exemple, où les gisements de sels d'alumine existaient en abondance, le tannage minéral (à l'alun ${ }^{16}$ ) prédominait. Les peaux et pelleteries blanches ou colorées des Egyptiens, des Babyloniens, des Hittites, étaient traitées avec de l'alun, minéral très recherché et largement commercialisé depuis l'Antiquité. Bien que la combinaison collagène-alun ne soit pas d'une grande stabilité chimique et fournisse un cuir très sensible aux effets de l'humidité, les tanneurs ont remédié à ce défaut en incorporant, par exemple, des matières grasses entre les fibres. Mais l'alun a des mérites incomparables. D'une part, il assure une blancheur éclatante aux peaux ou bien facilite la fixation des teintures (blancheur et couleurs vives étaient des qualités très valorisées au regard, notamment, des couleurs foncées des cuirs tannés végétalement). D'autre part, le tannage à l'alun est une technique plus rapide et moins complexe que le tannage végétal. Ce sont surtout les peaux légères des caprins et des ovins qui constituent la matière première du tannage à l'alun. Celui-ci, en France, fut principalement pratiqué par les Mégissiers (appelés aussi Blanchers) ${ }^{17}$ et les Peaussiers-Teinturiers-pareurs des peaux mégies fort en vogue jadis dans le domaine vestimentaire. Notons, à titre de fait peu exploré, que les nombreux extraits végétaux employés en tant que colorants pour leur action tinctoriale devaient très probablement posséder en outre un certain pouvoir tannant sur la peau traitée à l'alun.

Tannage à la graisse

Le tannage à la graisse était surtout fréquent dans les zones septentrionales. Il consiste à faire pénétrer dans la peau diverses sortes de matières grasses telles que l'huile de poisson ou de mammifères marins de préférence, mais aussi de la cervelle, de la moelle, du beurre, des jaunes d'œuf, etc., pour la transformer en cuir imperméable, résistant et moelleux ${ }^{18}$.

30 C'est une technique qui implique un gros travail mécanique (foulon nage, palissonage) et assure, entre autres, le relâchement des fibres (obtenu parfois au moyen de la mastication) et qui garantit également une certaine augmentation de la température, indispensable pour provoquer et accélérer le processus chimique de l'oxydation responsable de la transformation de la peau en cuir.

31 Le tannage à la graisse est souvent combiné avec le tannage à la fumée (chez les Indiens d'Amérique du Nord, par exemple). C'est un procédé très répandu dans les aires géographiques où le tannage végétal n'a pas joué un rôle significatif, comme en Asie centrale et en Extrême-Orient (chez les Mongols, les Tatars, les Chinois, etc.).

En France, le tannage à la graisse a reçu le nom de chamoisage. Ce procédé, souvent lié à l'activité des Mégissiers, produit une peau chaude et veloutée, « effleurée ${ }^{19}$, propre à l'usage corporel. Au XVIIe siècle, cette technique a acquis une importance essentielle pour l'équipement de l'armée (surtout de la cavalerie) qui consommait de grosses quantités de buffleterie, peaux de buffle tannées à l'huile.

Tan ou alun : l'exemple du cuir de Cordoue

33 En France, dans la longue période qui court de la naissance des premières corporations du cuir (XIIe-XIVe siècles) à leur disparition ou leur transformation en industrie du cuir (fin XIXe-début XXe siècles), nous avons pu distinguer deux grands axes technologiques coexistants : le tannage végétal, pratiqué par les Tanneurs, et le tannage minéral (à l'alun), principalement œuvre des Mégissiers ${ }^{20}$.

La tannerie - du fait de sa production (notamment de cuirs particulièrement forts et résistants) indispensable à de nombreux secteurs de l'économie- est depuis longtemps 
considérée comme la branche prépondérante de l'industrie du cuir. On l'a même souvent confondue avec toute cette industrie, en reléguant les autres techniques à une place secondaire. Or, on peut avancer, après analyse de la répartition des métiers du cuir, que la prétendue prédominance du tannage végétal devrait être remise en cause.

Les données nous amènent à constater une certaine alternance des deux techniques, suivant l'époque, les besoins, ou selon la disponibilité de tel agent tannique ou telle matière première. Nous passerons au crible l'indéniable importance du tannage à l'alun à travers deux exemples d'emprunts significatifs : la préparation du cordouan (dans la période de la fin du XIVe siècle et première moitié du XVe siècle) et le hongroyage (XVIIe-XVIIIe siècles), tous deux ayant marqué le paysage industriel de leurs époques respectives.

Avant l'introduction du tannage au chrome à la fin du XIXe siècle, l'alun fut l'unique agent tannique minéral, souverain pour la préparation massive de la peausserie et de la pelleterie-fourrure qui servaient de matériaux vestimentaires et domestiques à une population très importante. L'avantage essentiel de l'alun (outre la qualité des couleurs obtenues) consiste dans le fait que ce minéral assure un tannage nettement plus rapide, moyennant des techniques moins lourdes et moins complexes que le tannage végétal.

Parmi les nombreux corps de métiers qui consommaient abondamment de l'alun, il faut compter les Mégissiers, les Peaussiers-Teinturiers ainsi que la très importante corporation des Pelletiers-Fourreurs, mais aussi, jusqu'au XVIe siècle, celles des Cordouanniers et des Corroyeurs de Cordouan ${ }^{21}$.

38 Retrouver les préparateurs du fameux cuir de Cordoue en tant que tanneurs à l'alun est une découverte pour le moins inattendue et troublante, dans la mesure où elle va à l'encontre de toutes les informations véhiculées par les dictionnaires depuis le début du XVIe siècle. Dans le Dictionnaire de Nicot (1606), nous lisons la définition suivante : « Le cordouan est une espèce de cuir, qui est de peau de bouc ou de chèvre passé en tan, car celuy qui est passé en galle est appelé maroquin». Par la suite, la définition du cordouan comme produit "passé en tan", et même confondu avec le maroquin, devient une erreur assez répandue et reprise par d'autres dictionnaires ${ }^{22}$.

39 Cette confusion relative au caractère technique du cuir de Cordoue que nous avons eu l'occasion de relever une première fois au cours de la préparation d'un vocabulaire historique et technique du cuir ${ }^{23}$, nous a incité à pousser l'enquête plus loin en dépouillant bon nombre de documents :

40 - le Dictionarius de Jean de Garlande (XIIe siècle) désigne les « cordewaners » comme alutarii, c'est-à-dire travaillant à l'aide de l'alun;

41 - les Statuts des métiers, rédigés en 1350, mentionnent que la « ville de Paris avoit grande abondance de cordoën d'Espagne qui est le meilleur courroy des autres, eust esté ordonné que nul cordoën de Flandre n'y fust vendu pour ce que ceux de Flandrie estoient partie courroyez en tan "; cette interdiction concernant un article non conforme aux réglementations ou aux produits originaux vise les fraudes, dans une production corporative très strictement surveillée;

42 - les Statuts des «Mestiers des Cordoenniers" de Paris, à l'article 6 (de 1398) cité par V. Gay, nous confirment sans équivoque que le cordouan ne doit pas être tanné : «Nul cordouennier de Paris ne peult ouvrer de cordouan qui soit tanné car l'euvre serait faulse et devroit estre $\operatorname{arse}^{24} »$; 
43 - l'ouvrage Pratica della Mercatura ${ }^{25}$ nous apprend que sur les grands marchés de Provence (et notamment à Montpellier), au cours du XIVe siècle, on trouve en abondance du cordovano bianco en provenance de Valence et de Barcelone.

Le fait que le cuir de Cordoue soit apprêté selon la tradition du tannage à l'alun —savoir technique originaire d'Asie Mineure, emprunté et diffusé par les Arabes le long des côtes d'Afrique du Nord puis transmis, vers l'Espagne- n'a en soi rien de surprenant. La question la plus pertinente quant à ce type d'ouvrages est plutôt celle-ci : quand et pourquoi la technique du tannage à l'alun a-t-elle été supplantée par le tannage végétal?

Il nous semble nécessaire d'examiner d'abord l'intérêt technique et le poids économique de l'application de l'alun avec toute sa dimension historique, autrement dit, le contexte de l'industrie médiévale ${ }^{26}$. Les avis des divers auteurs concordent : au Moyen Âge, l'alun fut un des produits les plus importants du commerce. On allait le chercher en Syrie, en Égypte, en Grèce, mais surtout en Anatolie. Bien qu'indispensable à l'industrie du cuir, l'alun a tout d'abord été connu et recherché comme "un des principaux ingrédients des Teinturiers-Drapiers, qui servait à affirmer la couleur sur l'étoffe (mordançage)». "Sans alun, l'industrie textile s'arrêtait de tourner » ${ }^{27}$. L'importance de l'alun dans deux secteurs principaux - ceux du textile et du cuirexpliquerait déjà l'intérêt porté par le commerce médiéval à ce produit. Mais l'alun fut aussi largement utilisé dans d'autres domaines (la fabrication du papier, du verre, des bougies, etc.; il fut même employé en médecine contre les hémorragies). Dans ce contexte, il n'y a rien d'étonnant qu'à travers la possession des mines, les droits de son exploitation et de sa commercialisation, l'alun comptât pour une arme redoutable susceptible de déclencher des guerres économiques ${ }^{28}$.

Après la prise de Constantinople en 1453, l'alun oriental se fit plus rare sur les marchés occidentaux. La liquidation générale des intérêts génois en Orient eut pour conséquence une raréfaction brutale de l'alun et une montée sans précédent de son prix (de l'ordre de 400 à $500 \%$ en quinze ans dans la deuxième moitié du XVe siècle). Il devint urgent de remettre en activité les rares mines d'Occident (Pouzzoles, Ischia, Castille, Majorque, etc.), et surtout d'essayer de trouver de nouveaux sites. La découverte de la mine de Tolfa, près de Civitavecchia (propriété de l'État pontifical), très riche en alun, apporta enfin, quelques décennies plus tard, une solution à la crise, à tel point que cet " alun de Rome » inaugura une nouvelle ère économique ${ }^{29}$. En tout cas -et c'est cet aspect qui nous intéresse ici pour n'évoquer que les causes aboutissant aux changements techniques-, pendant un certain temps, la conjoncture économique défavorable a logiquement refoulé l'application de l'alun.

$\mathrm{Au}$ moins deux autres facteurs ont concouru à ce déclin et repoussé temporairement le développement du tannage minéral. Premièrement, l'existence incontestable d'un milieu technique européen essentiellement attaché au tannage végétal, dont la prédominance a certainement exercé une pression sur les autres procédés. Deuxièmement, le changement de la structure de la consommation dans la période de la guerre de Cent Ans -époque, également, de marasme économique-qui favorisait le recours aux cuirs forts et résistants issus du tannage végétal pour équiper l'armée, plus que l'utilisation des peausseries légères, liées à une certaine idée de richesse et même de luxe, dans la vie quotidienne.

Analyse d'un changement technique : tannage et hongroyage 

mesure où le changement s'opère cette fois-ci en sens inverse : un certain type de tannage à l'alun devait partiellement remplacer le tannage végétal, inapte à satisfaire les besoins accrus de l'époque.

Au cours des XVIIe et XVIIIe siècles, le tannage végétal pratiqué dans les fosses était devenu une technique particulièrement complexe, alourdie par la durée excessivement longue des étapes de fabrication et figée dans un immobilisme dû à l'inertie technique et aux réglementations immuables des corporations. Il fallait attendre en moyenne entre trois et cinq ans pour que le « tannage à cœur » des cuirs lourds et épais s'achève, tandis que la technique nouvelle des Hongroyeurs ${ }^{31}$, implantés en France au milieu du XVIIe siècle, proposait pour les mêmes produits (les peaux bovines) à peine quelques semaines de préparation.

«Il n'y a pas de cuir plus tôt fait que le cuir de Hongrie. Dans certaines provinces, on le fait en été dans l'espace de huit jours, mais c'est précipiter les opérations, on doit y mettre au moins quinze jours, souvent il faut même deux mois pour l'amener à sa perfection [...]. Le cuir de Hongrie parut mériter d'être favorisé [...] [car] malgré la promptitude de l'opération, ce cuir a des avantages considérables sur le cuir fort tanné.»

Ces quelques phrases du savant encyclopédiste Jérôme de Lalande nous introduisent au cœur du problème le plus crucial du tannage : le facteur du temps investi dans les opérations.

Pour mieux saisir l'importance de ce changement technique - précurseur d'une longue série d'innovations et d'emprunts intervenus au cours du XVIIIe siècle-, nous proposons en annexe une comparaison des deux procédés. Notre objectif est double: d'une part, en retraçant les principales étapes de la chaîne opératoire, faire ressortir la dimension technique des changements qui interviennent dans un processus de travail complexe, le tannage; d'autre part, mettre l'accent sur les nombreux dangers et seuils critiques qui marquent le cours des opérations et rendent l'activité des tanneurs extrêmement périlleuse. Ce dernier aspect n'est pas à négliger, car ces nœuds de difficultés techniques, entraînant des risques considérables, étaient souvent les véritables déclencheurs des changements et des innovations.

Nous tenons également à mettre en relief le caractère empirique du savoir-faire impliqué dans le tannage d'autrefois, fondé sur le rapport très intime qui liait les tanneurs à leur matière d'œuvre organique particulièrement instable. Sollicitant les cinq sens et faisant travailler le corps entier, il mobilisait un savoir corporel et perceptif considérable. Cette activité éprouvante exigeait une importante force physique et la capacité d'exécuter des gestes mesurés et souvent délicats, mais aussi l'aptitude à faire des observations nuancées pour déchiffrer dans l'aspect de matériaux changeants certains indices à peine lisibles des étapes non contournables dans la transformation de la matière.

Pour reconstituer les chaînes opératoires du tannage et du hongroyage, nous nous réfèrerons toujours aux volumes de Lalande (1762-64), dont les études détaillées et savantes furent commandées par l'Académie des Sciences afin que les «arts de la physique [...] pratiques, pleins d'esprit et d'invention, mais inconnus, soient tirés des ténèbres ». Grâce à ces mémoires, on possède un "condensé » des savoirs techniques qui remontent à la deuxième moitié du XVIIe siècle ${ }^{32}$, puisque Jérôme de Lalande a utilisé -outre ses enquêtes sur le terrain- des manuscrits non imprimés de 
l'Académie, en effectuant aussi quelques voyages à l'étranger afin de faire des comparaisons entre les procédés similaires.

La richesse des données d'ordre technique et la qualité de l'explication des procédés, reflétant aussi bien la position des praticiens que l'état de la science, amènent à reproduire plusieurs passages des textes de J. de Lalande, ce que nous avons fait d'autant plus volontiers que son langage est vivant et riche des expressions propres aux tanneurs (voir annexe).

Tendances techniques et innovations

"Accélération, freinage : la technique est l'un et l'autre de ces processus, souvent l'un puis l'autre; elle pousse en avant la vie des hommes, aboutit à petits pas à de nouveaux équilibres... puis s'y maintient longuement, car elle stagne ou progresse imperceptiblement... d'une innovation à une autre. » (Braudel 1979, tome $1: 486$ )

L'examen des chaînes opératoires respectives des deux techniques dominantes et concurrentes autorise la mise en parallèle d'opérations suffisamment similaires pour faire ressortir ce qui varie dans le processus de travail et évaluer également les causes intrinsèques des changements techniques. Leur impact, cependant, ne s'arrêtait pas là : il impliquait également les germes des innovations et des inventions à venir; il marquait la naissance des grands courants des tendances techniques (Leroi-Gourhan 1971 : 336-339) qui allaient affecter toute l'industrie du cuir dès la fin du XVIIIe siècle. Bien que la période qui précède la Révolution industrielle soit souvent interprétée comme "insignifiante " du point de vue du progrès technique, son apport, loin d'être négligeable, revêt un caractère tout à fait particulier: "[c'est] une période de transition fondamentale » (Daumas 1965, tome II : v-vii).

En dépouillant des ouvrages du XVIIIe siècle, nous avons pu déceler quelques tendances très significatives bien que peu «visibles » car sous-jacentes aux grandes structures relativement immobiles de la technologie de l'époque. Tous les manuels qui traitent de l'innovation dans le domaine du tannage s'accordent pourtant pour affirmer que c'est seulement à la suite du développement de la recherche scientifique - notamment des progrès de la chimie - et de l'introduction des machines (la mécanisation du travail manuel), à partir du milieu du XIXe siècle, quc commence la lente « ascension » vers la modernisation et l'industrialisation du travail des tanneurs, jugé jusqu'alors empirique et archaïque. Travail très peu rentable de surcroît, qu'on a pu caractériser ainsi : les capitaux des tanneurs étaient ensevelis dans les fosses et on ne pouvait ni les toucher ni les mobiliser pendant de longues années.

La personnalité marquante de quelques inventeurs et les effets d'un regain de ferveur dans la recherche des moyens de réduire la longue durée du tannage, ainsi que la découverte de nouveaux agents tanniques, ont été la source d'innovations assez bien connues et documentées; en revanche, les mouvements qui s'opéraient en profondeur dans les processus du travail (non distingués par le nom des inventeurs) et dont émergeaient des solutions techniques souvent considérables, sont des faits très peu étudiés et encore moins valorisés. L'un des objectifs de notre analyse des chaînes opératoires était justement de désigner les principaux facteurs de risque de telle ou telle opération, et par là, de comparer les différentes solutions techniques (voir annexe).

Nous tenons maintenant à récapituler quelques tendances techniques et aussi certaines innovations précoces qui préfiguraient une transformation structurale de toute l'industrie du cuir. 
Quantité et qualité du tan l'objet d'observations (et d'expérimentations pratiques).

«En Angleterre (à Oxford), on tanne dans l'eau même [sic]... et non pas dans une écorce presque sèche comme on le fait en France. La méthode des Anglais de tanner dans l'eau d'écorce est peut-être la source de l'avantage considérable que les cuirs d'Angleterre sont réputés avoir sur les nôtres » [...] «L'eau qui tient en dissolution les parties les plus pénétrantes et les plus stiptiques du tan, doit les pénétrer plus facilement et plus intimement que la poudre ou la boue d'écorce.» (Lalande 1762-64)

Cette remarque est particulièrement importante, car la reconnaissance du rôle de l'eau pour «dissoudre» les parties les plus actives de la matière tannante préfigure une invention capitale : la préparation des extraits tannants, qui va rendre possible aussi bien l'accélération des procédés ${ }^{36}$ que l'allègement du stockage et du transport des divers types de tanins en provenance de pays lointains. Cette invention ne date que de la fin du XIXe siècle...

Jus d'écorce

Pour économiser sur la grande quantité d'écorce appliquée dans les fosses, J. de Lalande proposa une solution technique qu'on pourrait qualifier d'innovante dans le processus du tannage lent : la réutilisation du jus des tannés, méthode qui préfigure l'extraction du tanin. Voici la description qu'en donna le savant:

«Pour préparer le jus de tannée, on ramasse la vieille écorce (dans laquelle les cuirs ont séjourné en seconde ou troisième poudre), on puise également le liquide qu'elle contient. On pourrait alors ménager dans un coin de la fosse un puisard pour y introduire une pompe. On puiserait par ce moyen le liquide filtré au travers de la 
tannée. Ces filtrations réitérées seraient un moyen sûr de tirer tout le parti possible de cette écorce [...] et on gagnerait beaucoup de temps avec cette méthode.» (Lalande 1762-64) chausser annuellement nos armées, on a besoin de 170000 peaux de bœuf, 100000 peaux de vache, 1000000 de peaux de veau ». Il était impossible d'attendre plusieurs mois, voire jusqu'à deux où trois ans, que ces peaux soient tannées. Une première alerte -contre la lenteur du tannage- fut provoquée par les guerres de la Révolution. L'armée devenait nationale et son équipement réclamait une quantité prodigieuse de cuir. En 1794, le chimiste et industriel Armand Seguin développa un procédé de tannage «ultra-rapide» (en trois semaines) en reconnaissant le rapport essentiel entre la concentration $\mathrm{du}$ jus tannant et la rapidité du tannage. II a également considérablement réduit la durée du travail de rivière.

Cependant, en temps de paix, ces inventions furent oubliées. Après la Révolution et les guerres napoléoniennes, les vieilles méthodes artisanales reprirent le dessus et un nouvel équilibre s'établit entre la demande sociale et la production traditionnelle. Guerre et paix feront encore l'arbitrage, au début du XXe siècle, quant aux méthodes de tannage à appliquer. Le "Cahier des charges de l'Armée Française " préconise les distinctions suivantes, en 1915, en matière de qualité des fournitures militaires :

« En temps de paix, par suite des besoins relativement faibles... et de la nécessité de conserver plus longtemps des approvisionnements en cuir, l'Administration Militaire avait imposé à ses fournisseurs un cahier des charges qui n'admettait que l'emploi de cuir tanné au tannage lent à l'écorce. En temps de guerre, la demande est trop grande pour qu'on puisse conserver les mêmes exigences. Le renouvellement incessant des objets en cuir permet une plus grande tolérance. Pour cette période, et pour ce1le-ci seulement, on admet donc dans la fourniture des cuirs obtenus par des tannages végétaux plus ou moins accélérés. Toutefois, il 
convient de ne pas perdre de vue que la qualité du cuir dépend essentiellement du mode de tannage appliqué. » (Passot 1923 : 46-47) concernant aussi bien la matière d'œuvre - la peau- que la technique qui la transforme et la change dans le temps en un matériau de base -le cuir. Nos remarques finales s'articuleront selon trois aspects du temps : la durée, le rythme et les saisons.

La longue durée des opérations était essentiellement due au fait qu'il fallait « laisser le temps agir" presque comme dans un processus naturel, en introduisant progressivement des matériaux divers comme agents responsables des changements. Le caractère très peu "dynamique" des procédés étant l'une des causes de cette lenteur, on y remédiait par l'action de tel ou tel travail mécanique (foulonnage, palissonnage...).

Le rythme des opérations était souvent déterminé par le recours à une alternance entre les phases actives - pendant lesquelles les agents chimiques en contact avec les peaux agissaient directement- et les phases de retrait -où l'on retirait celles-ci pour les laisser « reposer ». Il s'agissait d'un découpage du temps « savant» (ou non) dont le but était de prévenir les effets nuisibles des agents trop « agressifs » sur une matière assez vulnérable.

Les saisons étaient largement reconnues comme facteurs actifs au cours des fermentations, par exemple, car la durée de telle ou telle phase était sensiblement modifiée selon la saison, hivernale ou estivale, par la différence de température de l'air ambiant ou de celle de l'eau.

Dans l'ensemble, on serait tenté de considérer, sous un angle plus "philosophique ", que le tannage arrête l'effet du temps sur la matière (irréversibilité). Mais le temps ne s'arrête pas une fois le tannage achevé et le cuir obtenu. Si la peau est un tissu vivant qui enregistre dans son aspect externe, mais aussi dans sa structure, les «signes» du temps, le cuir -bien que pérennisé et quelquefois même "cuirassé» contre les altérations- est lui-même un matériau organique qui continue d'entretenir des échanges avec le milieu extérieur et reste inéluctablement voué à une dégradation irréversible.

Compte tenu, précisément, de cette vulnérabilité du cuir face aux nuisances du temps qui passe, il serait souhaitable de se préoccuper, à une plus grande échelle, de la protection de cette part du patrimoine et d'entreprendre une recherche plus systématique.

\section{BIBLIOGRAPHIE}

Balfet, Hélène (ed.)

1991. Observation de l'action technique. Des chaînes opératoires pourquoi faire? Paris : Éditions du CNRS.

Techniques \& Culture, 38 | 2002 
Braudel, Fernand

1979. Civilisation matérielle, économie et capitalisme XVe-XVllle siècles. Tome 1. Paris : Armand Colin.

Daumas, Maurice

1965. Histoire Générale des Techniques. Paris : Presses Universitaires de France.

Delumeau, Jean

1962. L'Alun de Rome, XVe- XIXe siècle. Paris : Éditions SEVPEN.

Franklin, Alfred

1987. Dictionnaire historique des Arts, Métiers et Professions exercés dans Paris depuis le XIIIe siècle. Marseille : J. Laffite (1ère édition Paris/Leipzig, 1905-1906).

Gansser, Auguste (Dr)

1951. «L'évolution du tannage primitif », Cahiers Ciba 34 : 1153-1184.

Gay, Victor

1887. Glossaire archéologique du Moyen Âge et de la Renaissance. Paris : Librairie de la Société bibliographique.

Lalande, Jérôme (de)

1762-64. L'Art du tanneur. L'art du hongroyeur. L'art du corroyeur. L'art du mégissier. L'art du chamoiseur. L'art de faire le parchemin. L'art de faire le maroquin. Paris : Dessaint et Saillant (10 fascicules en un volume).

Le Goff, Jacques

1977. « Métiers licites et métiers illicites », in Pour un autre Moyen Âge. Paris : Gallimard.

Leroi-Gourhan, André

1971. L'Homme et la matière. Paris : Albin Michel.

Passot, Émile

1923. Les Cuirs employés dans l'armée française. Paris (5ème édition).

Piersat, Jean

1942. Voyage dans le pays du cuir. Paris : Éditions Lang.

Savary des Bruslons, Jean

1723. Dictionnaire universel de commerce. Paris : Jacques Estiennes.

\section{ANNEXES}

\section{Annexes}

Description des divers processus techniques qui mènent de la peau brute au cuir (intégrant des citations de Lalande 1762-64 : sauf indication contraire, c'est toujours nous qui soulignons).

I. Opérations préliminaires du tannage Conservation

Les peaux qui ne sont pas immédiatement utilisées après l'abattage des animaux doivent être conservées afin de prévenir une détérioration rapide (en quelques heures) et irréversible, favorisée par la forte teneur en humidité (jusqu'à $75 \%$ ) des peaux vertes. L'élimination de l'eau peut freiner le phénomène. On y parvient soit en faisant sécher la peau, soit en la saupoudrant de sel, ce qui assure un double effet : action bactériostatique et drainage des tissus. Les tanneurs employaient généralement les 
deux méthodes combinées (salage, séchage), par « crainte de corruption de leurs peaux ». Les peaux salées (avec du sel de morue ou du sel mêlé d'alun) étaient mises en pile pour « donner au sel le temps de pénétrer » (8 jours en été, 15 jours en hiver). Les hongroyeurs, par contre, utilisaient exclusivement des peaux fraîches qui « conservent toute leur force », puisqu'elles «n'ont pas le temps de fermenter et de s'échauffer », c'est-à-dire de s'altérer en attendant les opérations suivantes souvent différées. L'avantage considérable de l'utilisation des peaux fraîches par les hongroyeurs consistait d'une part à garder intacte la structure fibrillaire (sans affaiblissement ou altération du tissu dermique), et d'autre part à économiser aussi bien des agents de conservation que du temps précieux.

Lavage/Reverdissage

«Comme le lavage est une opération qui revient sans cesse dans l'art du tanneur, il s'ensuit qu'une tannerie doit être établie au bord de l'eau et s'il se peut, d'une eau coulante.»

La désignation " travail de rivière ", qui concerne toute une série d'opérations préliminaires du tannage (lavage, reverdissage, pelanage, débourrage, écharnage, confitage), souligne l'importance de l'eau tout au long de la chaîne. La nature et l'état de la peau brute (salée ou séchée) sont des facteurs déterminants dans le choix du procédé de nettoyage : lavage et/ou reverdissage. Les tanneurs appliquent un simple lavage « lorsque les cuirs en poil [les peaux brutes] que l'on veut habiller sont verds, c'est-à-dire qu'ils conservent leur humidité naturelle », pour « les désaigner, les nettoyer du sang et des ordures ».

Quand les « cuirs » sont secs (salés et séchés) on a recours au reverdissage, qui est un trempage prolongé dans l'eau, alterné avec de fortes actions mécaniques : l'étirage (ou craminage) des peaux sur le chevalet avec un couteau émoussé (herbon); le foulage (à la force des pieds); le décrottage (avec un couteau « demi-rond »); le rinçage (agiter les peaux dans l'eau à la force des bras); et enfin, l'égouttage (sur des perches). J. de Lalande observe dans certains endroits l'utilisation d'une innovation : les moulins à fouler qui accélèrent le travail.

Durée et dangers de l'opération chez les tanneurs

« On laisse tremper les peaux jusqu'à ce qu'elles soient soulées d'eau, c'est-à-dire jusqu'au point où l'on commence à craindre la corruption. [...] Cependant il $\mathrm{y} a \mathrm{un}$ terme [...] on en juge par l'odeur ».

Suivant l'origine et l'état des peaux, ainsi que la qualité de l'eau et la température de la saison, la durée de l'opération variait de 1 à 4 jours. Les hongroyeurs n'appliquaient aux peaux fraîches qu'un lavage (nettoyage), évitant ainsi les risques majeurs tels que la putréfaction et s'épargnant le travail physique que constituent la manutention des peaux lourdes (30 à $50 \mathrm{~kg}$ pour une peau de bœuf) et les opérations mécaniques. La durée du nettoyage chez les hongroyeurs ne dépassait pas les 24 heures.

Pelanage ou travail de la chaux

Chez les tanneurs, « pour disposer les cuirs à être pénétrés par le tan, il fallait les faire enfler et en dilater les pores. De cette opération dépend le succès du tannage; un cuir ne saurait être bien tanné s'il a été mal préparé dans les pleins ${ }^{38}{ }^{\prime}$. Un vieil adage des tanneurs dit d'ailleurs : « ce qui pelane, tanne ». 
L'importance capitale de cette opération s'explique par son objectif complexe. Le pelanage est une action chimique pratiquée dans des bains de chaux éteinte à l'eau, au cours de laquelle l'agent chimique provoque une dissolution partielle des kératines constitutives de l'épiderme et des poils, tour en ménageant l'intégrité du tissu dermique qui ne subit qu'une légère dégradation. Cette dégradation - si l'action est bien maîtrisée- exerce une influence déterminante sur les propriétés physiques du cuir en augmentant sa souplesse et en améliorant la réactivité du collagène du derme à la matière tannante. " Dans tous les pays où l'on fait du cuir à la chaux, on a plusieurs sortes de pleins (morts, faibles et vifs) dans lesquels chaque cuir passe successivement en l'espace de dix, douze ou quinze mois ». On appelle pleins (dont le nombre varie de 3 à 12) « des grands creux pratiqués en terre qu'on remplit de l'eau de chaux ». Le travail des pleins est soumis à un rythme d'alternance du contact actif des peaux avec la chaux, qu'on appelle abattre (pendant une durée de 8 jours), et de la mise en retraite (les peaux sont rangées les unes sur les autres hors de la chaux, pendant 8 jours également). Le caractère progressif de ce travail est très important car il faut éviter le risque majeur : brûler la matière. La première étape de cette longue opération se déroulait dans les pleins morts (une vieille eau de chaux qui avait déjà « jeté son feu ») et avait pour but de préparer les peaux à l'épilation.

L'action chimique de la chaux est suspendue par deux opérations mécaniques : le débourrage et l'écharnage. Une fois qu'on avait débarrassé la peau de l'épiderme, des poils et de l'hypoderme, le travail de la chaux -dans des pleins faibles, puis vifsdevait se poursuivre encore pendant une période plus ou moins longue (de 4 à 12 mois) en alternant l'abattage et la mise en retraite. Danger et inconvénient de la longue durée du pelanage sont formulés dans cette critique de J. de Lalande : « Il nous parait que le grand nombre des pleins ne sert à rien et produit une dépense inutile [...]. Le cuir ne peut gonfler que jusqu'à un certain terme, au-delà duquel il ne fait plus que se brûler [...]. La chaux est corrosive, elle brûle la substance du cuir au point qu'on la voit souvent se déchirer. Le tan ne saurait réparer les fibres à moitié détruites, il ne peut que fortifier celles qui sont entières [...]. Cette méthode est la moins bonne de toutes celles que nous avons à décrire ${ }^{39}:$ L'usage de la chaux est certainement une des causes du discrédit de nos manufactures. »

La critique virulente du savant auteur de L'Art du tanneur mérite qu'on s'y arrête, car elle vise une question qui est sans doute l'un des nœuds de la difficulté technique du tannage. La problématique complexe du pelanage a représenté un facteur important de la recherche d'autres méthodes qui aboutirent, au cours du XVIIIe siècle, à une série d'emprunts et d'innovations.

Les hongroyeurs, quant à eux, n'employaient aucun produit chimique pour épiler leurs peaux. L'ébourrage et l'écharnage consistaient pour eux en un travail mécanique, le rasage. Ils le pratiquaient sur le chevalet à l'aide d'un couteau spécial, la faux, outil très aiguisé qui rendait l'opération efficace mais aussi délicate, cependant que le tanneur avait recours à un outil émoussée ${ }^{40}$. Pour prévenir le risque majeur -couper la peau-, les hongroyeurs avaient pour méthode de faire une couche, c'est-à-dire qu'ils couvraient le chevalet avec plusieurs peaux déjà échamées et superposées, « pour que la souplesse du fond puisse prêter aux inégalités de la peau et ne pas résister au couteau qui la couperait infailliblement ». Le travail préliminaire des hongroyeurs s'arrêtait là, ayant éliminé de manière purement mécanique les parties non utilisables de la peau brute, matériau de base qui de ce fait n'était altéré ni par la conservation ni 
par l'effet corrosif de la chaux. La simplicité et l'efficacité de ce type de travail préliminaire permettaient aussi une plus grande rapidité, aboutissant à un gain de temps considérable pour l'ensemble des processus de transformation de la peau en cuir.

Travail de rivière

Pendant que les hongroyeurs commençaient la deuxième grande phase de la chaîne opératoire, l'alunage des peaux, les tanneurs continuaient encore une longue série d'opérations mécaniques au bord de la rivière pour nettoyer et adoucir la peau devenue rigide et desséchée par l'effet de la chaux. Afin d'empêcher que « la fleur des cuirs ne se casse ", ils appliquaient le quersage, le foulage à pied et aussi le travail sur le chevalet avec le couteau rond, qui consistait à « donner une façon » (côté fleur et chair de la peau), forts travaux mécaniques alternés avec le trempage (nettoyage) dans l'eau de la rivière.

Confitage

Comme le pelanage ne se bornait pas à détacher le poil et l'épiderme, car par une action chimique il ouvrait aussi le tissu dermique -en produisant un important gonflement du derme- la peau ne pouvait passer au tannage dans cet état. Il était nécessaire d'en éliminer les produits alcalins combinés au collagène et de neutraliser les peaux. Tel était le but principal de la dernière opération préliminaire, le déchaulage-confitage.

Le confit était une infusion de substances végétales (son, orge, genêt) et/ou animales (fiente d'oiseaux, crotte de chiens) qui provoquait des fermentations (acides ou alcalines) en présence de produits enzymatiques.

«Je trouve sur un ancien mémoire de M. des Billettes, écrit en 1965, une manière de préparer les peaux qui est assez remarquable, puisqu'elle est oubliée actuellement en France, mais non pas en Angleterre... Il faut prendre de l'eau fraîche, assez pour tremper les cuirs; y ajouter des genêts verts, pilés ou hachés, ou même de la fougère verte, de la fiente de chien, de poulet et de pigeon et laisser tremper le tout ensemble pendant deux fois vingt-quatre heures, il faut enfoncer les peaux là-dedans et les $\mathrm{y}$ laisser pendant deux jours; après cela les travailler sur le chevalet ». Il relève à Oxford une autre modalité technique très intéressante : « Les cuirs y sont trois semaines seulement dans les pleins et on les met pour huit ou quinze jours dans la fiente de pigeon. La fiente de pigeon ramollit les peaux que la chaux avait durcies, elle les dilate et les prépare à être tannées ».

II. Le Tannage proprement dit Tannage dans les fosses

" Tanner un cuir, c'est lui ôter son humidité naturelle, augmenter la force de ses fibres et rendre le tissu plus compact ». Le tannage des « cuirs forts » (les grands cuirs de bœuf) se faisait dans les fosses, c'est-à-dire dans des creux pratiqués dans la terre, ronds ou carrés, bordés en bois ou en maçonnerie. Le tan, écorce de jeune chêne moulue, réduite en poudre " astringente et dessicative »-qualités qu'on retrouve dans beaucoup d'autres plantes-, ne devait pas être gardé longtemps car il « perdait sa force ».

Le tannage dans les fosses « à trois écorces » était en France la méthode la plus répandue pour transformer les grandes peaux au moyen d'une technique archaïque et immuable, qui n'avait guère changé au cours des siècles et qu'on pouvait encore observer en milieu artisanal au début du XXe siècle. Ce type de tannage, qui consommait de grosses quantités de tan -100 à $140 \mathrm{~kg}$ d'écorce pour une peau de bœuf qui pèse elle-même environ $30 \mathrm{~kg}-$, demandait aussi beaucoup de temps dans la 
mesure où les peaux devaient séjourner dans les fosses pour une durée de 12 à 18 mois. L'opération technique (dans cette deuxième phase) était relativement simple et se divisait en deux séquences principales : la mise en fosse (ou « habillage ») et l'abreuvage de la fosse.

Mise en fosse

D'abord, on poudre les cuirs avec l'écorce de chêne et on les met en pile pendant 3-4 heures pour qu'ils commencent «à prendre le feu de l'écorce » avant d'être couchés en fosse. On met au fond de la fosse un bon demi-pied de tannée (de l'écorce qui a déjà servi en fosse); sur cette tannée, on étend de l'écorce neuve bien moulue et un peu humectée; sur cette poudre on étend un cuir, sur celui-ci une autre couche et ainsi de suite.

Abreuvage de la fosse

Quand tout l'habillage est ainsi couché en fosse, on met au-dessus de l'écorce neuve qui couvre le dernier cuir, un ou deux pieds de tannée que l'on foule avec les pieds pour «faire un chapeau » (c'est-à-dire que l'on place des planches sur cette tannée et qu'on les charge avec des pierres). Ensuite, « on abreuve la fosse d'eau claire; on en verse suffisamment pour que dans l'espace d'une journée elle ne soit pas totalement embue ».

Durée du tannage à trois écorces : J. de Lalande souligne qu'il a trouvé selon les régions de France des manières différentes (nous dirions des variantes techniques) dans la façon de pratiquer le tannage en fosse.

La première écorce -qui s'emploie sur la "fleur du cuir »-dure de 3 à 4 mois (pendant lesquels on ne touche pas aux fosses); la seconde écorce « se donne par chair » et dure 4-5 mois; la troisième se donne de nouveau côté fleur pour une durée de 5 à 8 mois. À chaque fois qu'on change d'écorce, on balaie chaque cuir, on le bat, on le secoue, «pour que la vieille écorce n'empêche point la nouvelle de jeter son feu dans le cuir ».

"Les tanneurs qui me paraissent les plus sincères et instruits conviennent qu'il faudrait laisser les cuirs dans l'écorce beaucoup plus longtemps qu'on ne fait en France, ils s'y prendraient plus de qualité et plus de force, plusieurs sont persuadés que ces excellents cuirs de Liège et d'Angleterre qui passent pour les meilleurs de l'Europe, y sont restés trois ans ou davantage. »

Tannage à l'alun

La méthode de tannage qu'on appelle « aluner les cuirs » n'était pas seulement une opération incomparablement plus rapide que le tannage dans les fosses (quelques semaines au lieu de quelques années), mais aussi beaucoup plus dynamique, car le processus chimique de la transformation des peaux se trouvait accéléré par de forts mouvements (actions) mécaniques, En effet, la méthode des hongroyeurs -le hongroyage- relevait de processus de travail plus complexes que celle des tanneurs, étant donné que cette technique mettait à contribution plusieurs sortes d'agents chimiques (alun, sel, suif) et la combinaison de deux types de tannage : le tannage minéral et le tannage à la graisse ${ }^{41} \mathrm{La}$ transformation de la peau en cuir dans les ateliers des hongroyeurs se divisait en quatre séquences principales : 1 ) alunage " en quatre eaux » (ou encuvage); 2) travail de grenier; 3) mise en suif; 4) flambage des cuirs ${ }^{42}$. Alunage

Dans une chaudière, on chauffe l'eau, l'alun et le sel à une température l'elle « qu'on ait peine à y tenir la main ». On pose les cuirs dans des cuves ovales (encuvage) et on y 
verse l'eau d'alun. Alors, un homme entre dans la cuve « nus-pieds » et « foule les cuirs à grands coups de talon ». On leur donne " trois tours ", c'est-à-dire qu'on fait aller trois fois d'une extrémité de la cuve à l'autre. Ces trois jours s'appellent la première eau, et il faut quatre eaux à chaque encuvage. Au total, on fait douze fois fouler les cuirs en trois encuvages : «Ce filtrage est très nécessaire au cuir de Hongrie pour faire pénétrer le sel et l'alun ». Après cette opération, fortement accélérée par le foulage, on laisse tremper les cuirs dans un baquet rempli d'eau d'alun pendant environ une semaine. Puis on les repasse en répétant une seconde fois le processus d'alunage d'une manière semblable à la première.

Travail de grenier

Les cuirs égouttés et séchés seront redressés par terre à l'aide d'une baguette fortement appuyée contre la chair du cuir, puis on les remet à sécher. Cette opération comporte une série d'actions mécaniques telles que rouler et piétiner les cuirs « pour les ouvrir et ramollir, afin qu'ils soient disposés à recevoir le suif ».

Mise en suif

"La mise en suif est la partie essentielle du hongroyage parce que la souplesse qu'elle assure au cuir est sa qualité principale ». L'introduction de la matière grasse dans le tissu dermique, sous l'effet de la chaleur, assurait un deuxième tannage (à la graisse) en complément du tannage (minéral) à l'alun qui, à lui seul, n'aurait pas pu garantir les propriétés physico-chimiques exigées des cuirs de fatigue (notamment une haute résistance à la traction, et l'irréversibilité du tannage). La mise en suif se déroulait dans une chambre hermétiquement close appelée étuve, qui devait garder la chaleur nécessaire à échauffer les cuirs pour qu'ils « jettent leur humidité ». L'étuve était équipée d'un fourneau s'allumant de l'extérieur et d'une chaudière qui pouvait contenir environ 160 livres de suif. Le feu bien allumé, les cuirs étaient placés par 30 bandes 43 (formant une venue) sur les perches, afin qu'ils s'échauffent. Dans la chaleur, « les cuirs jettent en forme de vapeur leur humidité (il y a des cuirs qui pleurent beaucoup) ». Ensuite, les cuirs sont installés sur des tables chair en l'air (avec les pores ouverts) et un ouvrier, à l'aide d'un gipon ${ }^{43}$, étend le suif autant de fois que le cuir « a besoin ».

Flamber les cuirs

Après l'opération de la mise en suif, les cuirs sont empilés et on les laisse ainsi reposer et boire leur suif, « deux ouvriers prennent alors les bandes de cuir et les passent sur la flamme du charbon pendant une minute environ. Cette chaleur ouvre les pores et les prépare à l'intromission du suif ». Finalement, les bandes de cuir sont posées sur une table (on les couvre avec une toile) et on laisse ainsi le suif pénétrer entièrement dans toute l'épaisseur du tissu fibreux de la peau. Ensuite, on met les cuirs à l'air libre pour les refroidir et pour qu'ils « reprennent leur fermeté ». Au bout de 2 ou 3 jours de « repos » en pile, la préparation du cuir de Hongrie s'achève.

III. Corroyage ou finition

Le cuir brut obtenu après le tannage, matériau appelé aussi cuir en croûte (d'après son aspect cartonneux), doit encore subir une longue série de traitements de finition destinés à lui conférer ses qualités définitives selon la destination finale. Le principal objectif du corroyage -en cas de cuirs forts tannés- est d'assurer la flexibilité, la résistance et la fermeté, au moyen de graissages (avec du suif, de l'huile, Je la cire, etc.) et par des opérations mécaniques. Le travail des corroyeurs était tellement complexe et 
diversifié que ceux-ci constituaienr (jusqu'en 1776) un corps de métier distinct de celui des tanneurs.

Le cuir hongroyé ne nécessitait pas l'opération du corroyage, car la mise en suif avait déjà assuré à elle seule les qualités exigées de ce type d'ouvrages : souplesse et résistance. «Le cuir de Hongrie parut mériter d'être favorisé car après avoir servi cinqsix ans, il conserve encore presque toute sa force... ce qui prouve le grand avantage de cette préparation et sa supériorité sur toutes les autres manières de préparer le cuir ».

\section{NOTES}

1. Le tannage est la désignation quasi exclusive de tous les tvpes de transformation de la peau en cuir : pratique linguistique qui pose le problème de l'imprécision du vocabulaire technique.

2. Milieu technique déterminant, qui a peu varié depuis l'époque de la fondation et de la spécialisation des corps de métiers du cuir (Xlile·XIVe siècles) jusqu'à leur disparition et leur transformation en une industrie « moderne » (de la fin du XVIIIe siècle au début $\mathrm{du}$ XXe).

3. À propos des métiers illicites, voir Le Goff (1977 : 93).

4. Au XIXe siècle par exemple, l'industrie du cuir occupait le troisième rang après la métallurgie et l'industrie du textile dans l'économie du pays, voir notamment le rapport Pelltereau à l'Exposition universelle de 1900, cité par Piersat 1942 : 11).

5. Du moins Jusque dans les années 1930, lorsque, suite à l'abondante production suscitée par la Première Guerre mondiale, la plupart des tanneries se mirent à investir dans le développement de leur entreprise.

6. Le tannage végétal dans les fosses durait de 2 à 5 ans, encore au XVIIIe siècle.

7. Nous avons appliqué le concept de la chaîne opératoire « comme outil polyvalent d'observation, de description et d'analyse des processus techniques » (Balfet 1991 : 11).

8. « Nom que les Tailles de 1292 et de 1500 donnent aux cordonniers... qui devaient leur nom à l'espèce de cuir qu'ils employaient, le cordouan » (Franklin 1987, t. $1: 204$ ).

9. Le nombre des maîtres cordouanniers est parmi les plus élevés selon la Taille de la Ville de Paris : 275 en l'an 1300 ! (Franklin 1987 : 203).

10. Il est important de souligner qu'en dépit d'une industrie du cuir qui se développe, se diversifie et se diffuse très tôt en France, le rendement de la production locale s'avère souvent insuffisant et notamment pendant les périodes de guerre (l'armée consommant énormément de cuirs d'équipement), mais aussi en temps de paix en cas de pénurie ou de prix élevés de certaines matières premières, par exemple. Pour combler le manque et pour compenser les aléas d'une production lente, peu efficace, lourdement dependante de nombreuses contraintes extérieures et, de plus, compromise par les dangers inhérents à la fabrication, on fait donc appel aux échanges commerciaux qui fournissent materiaux bruts, agents tanniques ou produits finis. 11. Sujet développé dans une communication présentée au colloque d'Antibes (octobre 2001).

12. Terme et définition cmployés par le Dr Augusle Gansser (1951:1153-1184).

13. Cette distinction, proposée par A. Gansser (1951), est particulièrement importante du point de vue de la conservation et de la restauration des objets en cuir.

14. Nous tenons à souligner l'importance du thème de la grande diversité des matériaux utilisés au cours des tannages, car il relève, entre autres, d'une vaste gamme 
de savoirs botaniques, zoologiques el minéralogiques mis en œuvre, sujet qui mériterait également d'être exploré par des études ethno-scientifiques.

15. «Cette méthode du cuir au sippage (ou cuir à la danoise) consiste à tanner les cuirs en deux mois... on la pratique surtout en Bretagne » (Lalande 1762-64 : 89-90).

16. Sulfate double d'aluminium et de potassium, l'alun était aussi désigné comme « sel des Hittites », ou « alun (sel) de Roche » d'après la ville de Roche (Rocca), près de Smyrne en Syrie, où se situait un important gisement d'alun.

17. « Le mégissier est celui qui prépare les peaux blanches ou peaux mégies, avec la chaux, l'alun, le sel, le confit et la pâte » (Lalande 1762-64 : 199). (La pâte étant un mélange d'eau, d'alun, de sel, de farine, de jaunes d'œuf et d'huile.)

18. Il faut souligner que diverses matières grasses étaient aussi abondamment utilisées au cours du corroyage-finition des cuirs tannés végétalement, ou encore par des mégissiers ou des hongroyeurs.

19. La fleur de la peau, la couche la plus dense, la plus difficile à tanner, est enlevée, ce qui donne un aspect fibreux, pelucheux aux peaux chamoisées.

20. Fait curieux : en dépit des changements technologiques radicaux intervenus depuis lors, l'industrie française du cuir est encore aujourd'hui divisée en ces deux branches, la Tannerie et la Mégisserie. «Le gros cuir et la petite peau relèvent de champs d'activité bien éloignés, qu'il s'agisse de leur technique ou de leur vocabulaire. » (Piersat 1942: 26).

21. La Taille de la ville de Paris, en 1500, énumère 30 maîtres-Tanneurs, 38 Mégissiers, 344 Pelletiers et 275 Cordouaniers. À titre de comparaison, le nombre des Drapiers à la même période n'est que de 56 à Paris (Franklin $1987:$ t. $1: 203,341$, t. $2: 475,679$ ).

22. Ainsi Savary (1723) définit aussi le cordouan comme " une espèce de maroquin ", quoique le même ouvrage cite le Tarif des douanes de 1706, qui distingue six sortes de courdouans, « marchandises du Levant » : les cordouans rouges d'Alep; les cordouans blancs; les cordouans de Smyrne; les cordouans de Chypre; les cordouans d'Italie; les cordouans en basane.

23. En tant que Chargée de mission de la Direction du Patrimoine, Inventaire Général (1988-89), pour l'élaboration d'un Vocabulaire technique du cuir et de la normalisation des termes anciens en relation avec les domaines des arts appliqués.

24. Arse (de ardre, arder ou ardoir) : brûlé.

25. Pegolotti : Pratica della mercatura (1340), p. 229, cité par V. Gay (1887).

26. Notre source principale est l'excellent ouvrage de J. Delumeau (1962).

27. Voir l'article sur l'alun dans l'Encyclopédie de Diderot et d'Alembert, cité par Delumeau (1962: 13-14).

28. Les principales mines étaient situées aux environs de Phocée, au fond du golfe de Smyrne; elles furent exploitées à grande échelle par quelques grandes familles génoises à partir de 1264. Ces carrières produisaient 14000 cantares, soit environ 700 tonnes d'alun par an en moyenne. Les mines passèrent entre d'autres mains, puis furent reconquises par la flotte armée de la fameuse association des marchands d'alun « Maona de Chio » en 1346 (Delumeau $1962: 16$ ).

29. Pie II a rapporté clans ses Commentaires la visite de la mine de Tolfa et le discours que lui fit Giovanni de Castro, aussitôt après la decouverte : « Aujourd'hui, je t'apporte la victoire sur les Turcs. Chaque année, ils extorquent aux Chrétiens plus de trois cent mille ducats d'or pour l'alun [...]. J'ai découvert sept montagnes tellement riches en alun qu'on pourrait en approvisionner sept mondes [...]. Maintenant, tu peux préparer la Croisade contre les Turcs, les mines te donneront les finances nécessaires ». À partir 
de 1463, Pie II interdit aux marchands chrétiens d'importer de l'alun oriental, et par la suite plusieurs papes ont brandi l'anathème envers le pays chrétien qui aurait osé rompre le monopole pontifical (Delumeau 1962).

30. « Le cuir de Hongrie est un cuir fort qui a passé dans l'alun et le sel et qui a été imbibé de suif » (Lalande 1762-64 : 299).

31. Les Hongroyeurs sont les préparateurs du cuir de Hongrie, qui est, selon l'Encyclopédie de Diderot el d'Alembert « une espèce de cuir qui tire son nom des Hongrois, qui seuls avaient autrefois le secret de le préparer. Il n'y a pas longtems que l'on connoit en France la manière de préparer le cuir de Hongrie. On prétend que ce fut Henri IV qui en établit la première manufacture; pour cet effet il envoya en Hongrie un tanneur fart habile nommé Roze qui, ayant découvert le secret, revint en France, où il fabriqua cette espèce de cuir avec beaucoup de succès » (1777-1780 : 555).

32. C'est en 1666 que " par ordre du roi et par les soins du grand Colbert, l'Académie des Sciences décida de faire étudier et décrire ces arts dans l'état où ils étaient en France » (Lalande 1762-64).

33. On appelle « pelard » le bois ainsi dépouillé qui « n'était plus bon qu'à brûler et même inférieur en qualité, car il brûle plus vite, en donnant beaucoup de flamme et peu de chaleur » (Lalande 1962-64).

34. Selon une statistique qui date de la veille de la Première Guerre mondiale, plus de 2000 tonnes d'écorce par an et par tannerie. « Il était à craindre de voir enfin manquer de bois en Europe, à cause de l'étonnante destruction qu'on ne cesse d'en faire pour les bâtiments, chauffage et pour les arts... » (Lalande 1762-64).

35. Le terme « travail de rivière » a survécu jusqu'à nos jours, tant la consommation en eu reste un aspect marquant du tannage.

36. Pour accélérer le tannage, les premières « étincelles » arrivent déjà en 1794, de la part d'un chimiste formé par Lavoisier, Armand Séguin. Processus formellement recommandé dans la période de la Révolution par la Convention Nationale « pour favoriser l'économie du pays » et surtout pour satisfaire les besoins accrus de l'armée et de la population. Seguin a reconnu le rapport primordial entre la concentration des jus tannants et la rapidité de leurs effets. Il a fondé des tanneries à Sèvres et à Nemours, où il a appliqué la nouvelle méthode, en produisant en 30 jours (!) des cuirs à semelles pour l'armée, « mais en employant aussi de l'acide sulfurique, il a vite fait des mauvais cuirs, cette aventure se termina en prison » et le procédé a été abandonné et oublié pendant presque cent ans.

37. Encore une remarque de Lalande : il se demande « s'il n'y aurait pas un avantage considérable à échauffer l'eau d'une fosse, car l'eau chaude dissout, ramollit bien mieux que l'eau froide ".

38. Le mot plein vient de peler. Le premier usage de la chaux étant de dégager la peau de son poil » (Lalande). De nos jours, on écrit plain et on parle de plamage, la plamée étant le blanc de chaux.

39. De Lalande décrit toute une série de techniques différentes pour épiler les peaux, qui n'utilisaient pas la chaux et dont la durée était considérablement raccourcie. Ainsi, la méthode anglaise (déjà citée) ou celles du cuir de Valachie ou du cuir de Transylvanie, appliquant toutes deux l'effet de la fermentation des céréales (blé, orge). 40. Les poils, déjà partiellement déracinés par l'action du pelanage, se détachaient assez facilement. 
41. Le tannage « mixte » ou « combiné » est une « invention » propre au XXe siècle pour obtenir une qualité supérieure dans l'industrie du cuir. Par exemple, on fait un pré-tannage minéral suivi d'un tannage végétal.

42. On peut se demander si l'usage du feu (pour flamber les cuirs) n'a pas en outre procuré un effet de tannage à la fumée.

43. C'est un paquet de grosse laine traversé par une poignée de bois.

\section{RÉSUMÉS}

Si la réduction de la longue durée du tannage végétal et la recherche des nouveaux agents tanniques constituaient la principale préoccupation des innovateurs de l'industrie du cuir dès le XVIIIe siècle, la devise des tanneurs français était encore au début du XXe siècle : « Pour avoir du bon cuir, il faut du tan et du temps ». Ces deux facteurs sont responsables de l'inertie technique : le tan (écorce de chêne) appliqué en grande quantité et le temps, excessivement long (deux à trois ans) investi dans les opérations de tannage, pour ce secteur-clé de l'industrie française, si peu étudié jusqu'ici. Par l'examen des diverses techniques de tannage et du rôle des emprunts techniques venant de l'étranger, l'auteur tente de dégager les principaux axes technologiques de la transformation de la peau en cuir.

Tan and time. Technical change and historical dimension of tanning in France (14th-18th centuries)

The reduction of the extreme length of vegetal tanning and the search for new tannic agents were the major preoccupations of the leather industry since the 18th century. However, even at the beginning of the 20th century, the motto of French tanners was still : « To get good leather, you need tan and time ». Two elements therefore were responsible for the technical inertia of this little-known key-sector of the the French industry : tan (oak-tree bark) used in great amount and time, excessively long (up to three years). Examining various tanning methods and the role played by the introduction of techniques from abroad, the author outlines the main technological axes of the conversion of raw hide into leather.

El tanino y tiempo. Cambio técnico y dimensón histórica en el curtido en Francia (S. XIVXVIII)

Si la principal preocupación de los innovadores en la industria del cuero, a partir del siglo XVIII, fue reducir la larga duración que comportaba el proces de curtido mediante productos de origen vegetal, e investigar acerca de nuevos agentes «tanínicos », la divisa de los curtidores franceses a principios del siglo XX aún era : «Pare conseguir buen cuero hace falta tiempo y tanino ». Ambos factores son responsables de la inercia técnica en este sector clave de la industria francesa que hasta ahora ha sido tan poco estudiado : el tanino (extraído de la corteza del roble) aplicado en grandes cantidades, y el tiempo excesivamente largo (de dos a tres años) que se invertía en las operaciones de curtido. A travès del examen de las diversas técnicas de curtido, y del papel desempeñado por las innovaciones técnicas que provenían del extranjero, la autora intenta desentrañar los técnicos principales de la transormación de la piel en cuero. 
INDEX

Mots-clés : France, cuir, savoir technique, tannage

Keywords : leather, tanning, technical knowledge

\section{AUTEUR}

EVA HALASZ-CSIBA

GDR 1201, Musée de l'Homme, Place du Trocadéro, 75116 Paris. 